\title{
APPROPRIATE ABDOMINAL IMAGING FOR THE EMERGENCY DEPARTMENT PATIENT
}

\author{
DANIELLE R. KNOTT* \\ Dalhousie University, Faculty of Health \\ Halifax, NS
}

\begin{abstract}
Abdominal x-ray series (AXR) and abdominal CT scans (ACT) are commonly performed to aid in the diagnosis for patients who present to the emergency room with abdominal pain. Patients commonly receive both an AXR and ACT, due to a lack of knowledge regarding imaging appropriateness among healthcare professionals who order these exams. A primary simple retrospective data-analysis was performed to understand the prevalence of how often both exams were ordered in three Nova Scotia emergency departments. A literature review was also conducted to compare the diagnostic accuracy of each diagnostic imaging modality. Several articles showed that patients who have an AXR also have an ACT that demonstrates an abnormal finding. Emergency department physicians are not reassured when abdominal $\mathrm{x}$-rays are negative and do not show abnormal findings, and as a result, a CT scan is also performed. Radiation dose must be considered when ordering multiple diagnostic imaging exams. A low-dose CT (LDCT) can be used to reduce the radiation exposure to the patient, while maintaining high diagnostic quality images. Image quality can be enhanced at a reduced radiation dose by using an image reconstruction technique such as adaptive statistical iterative reconstruction (ASIR). Understanding the most appropriate abdominal imaging modality for emergency department patients allows for fewer examinations being ordered and a reduction of radiation dose to the patient. When the most appropriate imaging is performed, a definitive diagnosis can be made and the best treatment can be provided to patients. This information can help to create an imaging appropriateness protocol for emergency departments. Additional research can help determine the cost differences between the two exams and the influence a protocol change could have on the emergency and diagnostic imaging departments.

Keywords: AXR - Abdominal x-ray series, ACT - Abdominal computed tomography scan, CT - Computed tomography, SDCT-Standarddose CT, LDCT - Low-dose CT, ASIR - Adaptative statistical iterative reconstruction $\mathrm{FBP}-$ Filtered back projection, $\mathrm{CTDI}_{\mathrm{vol}}-$ Volume computed tomography dose index
\end{abstract}

* Author to whom correspondence should be addressed: danielle.knott@outlook.com 


\section{INTRODUCTION}

Diagnostic imaging is a tool commonly used by hospital emergency departments that helps to determine the cause of a patient's symptoms. If a patient's history, physical examination, and laboratory testing does not identify the underlying cause of their symptoms, imaging is indicated as the next step in the diagnostic process (Cartwright \& Knudson 2015). Indications for patient's presenting to the emergency department that would require abdominal imaging include suspected bowel obstruction, abdominal pain, constipation, suspected bowel perforation, and foreign bodies within the abdomen (Bertin et al. 2019). Imaging for these clinical indications is either an abdominal x-ray series (AXR) and/or an abdominal computed tomography (ACT) scan. An AXR consists of two images: an image of the abdomen with the patient flat on their back and an upright abdominal image (or an image with them on their left side) to demonstrate free air and fluid within the abdomen (see Fig 1)

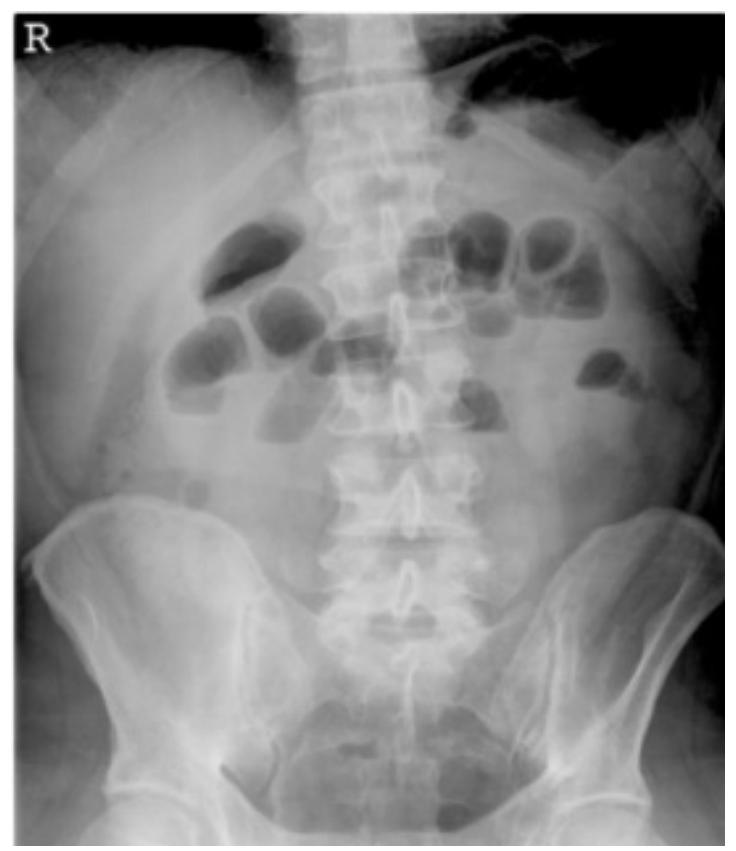

Fig 1 Abdominal x-ray (AXR) demonstrating the entire abdomen from the diaphragm to the symphysis pubis (Gans, Stoker \& Boermeester, 2012). 


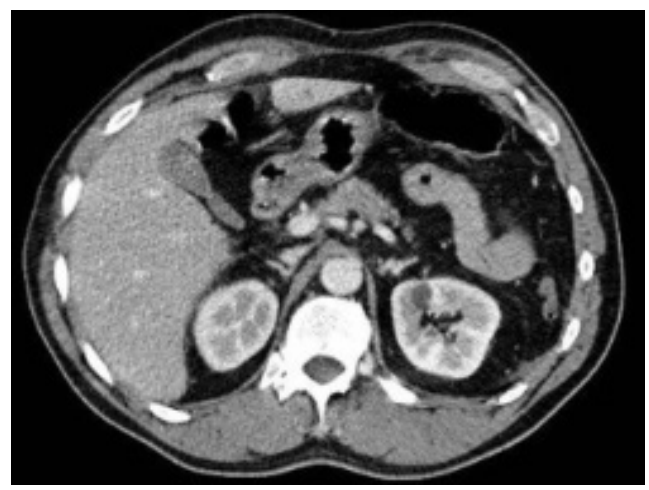

Fig 2 Abdominal Computed Tomography scan (ACT) demonstrating an image taken as the level of the kidneys and liver (Gans, Stoker \& Boermeester, 2012).

(Gans, Stoker \& Boermeester 2012). An ACT includes a low-dose x-ray called a topogram to plan the scan and includes anatomy from the patient's diaphragm to the symphysis pubis of the pelvis (see Fig 2) (Nguyen et al. 2011). The scan combines multiple x-rays from different angles around the patient and uses computer processing to create cross-sectional images. This creates a three-dimensional image of the patient's abdomen (Ngyuen et al. 2011). It can be difficult for the emergency room physician to determine the most appropriate abdominal imaging exam for a patient, due to a lack of knowledge that exists among physicians and health care professionals regarding which tests provide the best diagnostic performance (Bertin et al. 2019). This results in emergency physicians and other healthcare professionals, such as nurse practitioners, ordering both the AXR and the ACT to ensure that a definitive diagnosis can occur. Performing both exams results in an increase of radiation dose to the patient, so it is important to compare the diagnostic sensitivity of both imaging modalities to comprehend which is the most appropriate abdominal imaging examination. Increasing awareness and education regarding imaging appropriateness for the abdomen can help hospitals create imaging protocols that will minimize additional imaging exams for those emergency department patients who require abdominal exams. 


\section{METHODOLOGY}

\section{Primary Data-Analysis}

A primary retrospective simple data-analysis was performed through the picture archive computer system (PACS) to retrieve data from May 1-31, 2019.

\section{Eligibility Criteria}

A secondary literature review was performed. Articles eligible for this must adhere to the comparison of AXR and ACT for abdominal pain. Articles from the last five years will be considered to provide recent research. To maintain a high level of evidence, studies will be limited to retrospective cohort studies, literature reviews, prospective and systemic reviews.

\section{Sources and Search Strategy}

Three electronic databases were searched, including PubMed database, Google Scholar, and the Cochrane Library. A search example is described when using the PubMed database. The key search terms include abdominal $x$-ray, abdominal $C T$, radiation dose and abdominal pain. The dates were filtered to the past five years (2015-2020) and limited to randomized control trials, metaanalysis' and systematic reviews. Specifically, the results were narrowed by using the Boolean operator AND. The other databases were similarly searched. A secondary literature search was performed by reviewing references from relevant and appropriate publications.

\section{A RELEVANT ISSUE}

The Canadian Association of Radiologists (CAR) currently promotes the use of the Diagnostic Imaging Referral Guidelines (2012) to aid physicians in selecting the appropriate diagnostic imaging exam depending on the patient's symptoms. These guidelines are divided into thirteen sections depending on the area of the body and the appropriate indications (Canadian Association of Radiologists 2012). The recommended diagnostic imaging exam is "graded as being indicated for the patient, to be used for specialized investigation, not indicated initially, indicated only in specific circumstances or not indicated entirely", cited in the Canadian Association of 
Radiologists "Referral Guidelines at a Glance" (2012). The possible imaging exams include computed tomography (CT), magnetic resonance imaging (MRI), nuclear medicine, positron emission tomography (PET), and ultrasound (Canadian Association of Radiologists 2012). This resource is available to physicians and healthcare professionals, yet observations have been made that both AXR and ACT are being ordered in emergency departments, promoting the need to investigate the best and most appropriate diagnostic imaging modality for certain clinical indications.

\section{Data Collection}

A simple retrospective data-analysis of three emergency departments within the Nova Scotia Health Authority, including the Dartmouth General Hospital, the Cobequid Community Health Centre, and the Halifax Infirmary, was completed to investigate how frequently both an AXR and ACT were ordered by emergency room physicians during a one-month period. The relationship between sequential imaging, common indications, and suspected pathologies were reviewed. Excluded from this data collection for all emergency departments was ACT indicated for renal colic and hematuria. This was due to the fact that an imaging protocol is already in place for these indications within these emergency rooms (Nova Scotia Health Authority 2019). A limitation to this study includes the short collection timeline. An expansion of this timeline may be needed in order to elaborate and support results.

\section{RESULTS}

Emergency room physicians at the Dartmouth General Hospital ordered 86 AXR and 144 ACT during the study date, for a total of 230 abdominal imaging exams (PACS 2019). Thirty patients had both the AXR and ACT performed. The most common indications for abdominal imaging was abdominal pain. It is noted that the symptoms include nausea, vomiting, and constipation (PACS 2019). Location of the abdominal pain for each patient was detailed on the emergency room requisition provided to the diagnostic imaging department. The pain presented was between all four main abdominal quadrants. Common pathologies investigated were possible bowel obstruction and diverticulitis. The Dartmouth 
General Hospital emergency department demonstrated most, if not all, AXR being performed before the ACT. The most common finding on the AXR was air-fluid levels and a visualization of constipation (PACS 2019). A large number of findings within the reports for these AXR were normal or inconclusive, while the ACT for the same patient provided results that are "conclusive or highly suspicious of disease" (PACS 2019). It was common for the patients from this emergency department to have their AXR performed during the daytime diagnostic department imaging hours. The ACT was performed the following day. In a few instances for this emergency department, the AXR would report that the ACT was subsequently obtained and reported separately (PACS 2019). This could indicate that the AXR was not reported prior to the CT being ordered by the emergency room physician.

The emergency room at the Cobequid Community Health Centre requested $98 \mathrm{AXR}$ within the same month and $115 \mathrm{ACT}$, for a total of 213 abdominal imaging examinations requested (PACS 2019). Thirty-seven patients had both an AXR and an ACT. Similarly to the Dartmouth General Hospital emergency department patients, the most common indications for both abdominal imaging exams were also abdominal pain and constipation. There were some cases where a prominent history of abdominal pathology indicated both imaging exams, with pathologies such as Crohn's, ischemic gut, recent surgery, pancreatitis, and endometriosis being the most prevalent (PACS 2019). The Cobequid Health Centre emergency room physicians were suspicious of diverticulitis and small bowel obstruction when ordering both the AXR and ACT. While obtaining data for this emergency room, it can be noted that a majority of sequential exams on one patient are performed 3-4 hours apart, with the AXR completed first on the patient and the ACT a few hours later (PACS 2019). Within AXR reports of patient's who also received an ACT, very few radiologists suggested an ACT. There was one instance where the radiologist noted on an AXR report that a CT of the abdomen and pelvis was subsequently performed and to refer to the report for more details on the patient's condition (PACS 2019).

The Halifax Infirmary emergency room ordered 95 AXR and 217 ACT, for a total of 312 abdominal imaging exams (PACS 2019). Fifty-four patients received both scans. Although constipation as a symptom was not as common for Halifax Infirmary patients who 
received sequential abdominal imaging, abdominal pain with nausea and vomiting continued to be most common (PACS 2019). The most commonly suspected pathology was small bowel obstruction. Some other pathologies included bowel perforation, abscess, and incarcerated hernia (PACS 2019). Radiologists at this hospital were more likely to add in the report if they believed an ACT should also be performed, due to a suspicion evidenced on the AXR. There was one case that the radiologist believed there was a bowel obstruction on the AXR, and they alerted the emergency room physician to arrange for an ACT (PACS 2019). There were also some cases where the AXR and ACT were completed before the AXR report was completed (PACS 2019). See Table 1 for companions between hospital results.

\section{Data Interpretation}

A significant number of emergency room patients who present with abdominal pain received both an AXR and ACT in several Nova Scotia hospitals. A majority of emergency room physicians and healthcare professional have requested both imaging exams without the AXR report, which was the first diagnostic exam completed. One hypothesis could be that emergency room physician believes an $\mathrm{ACT}$ is necessary given the patient's symptoms. To properly perform an ACT, patients require oral preparation. Oral preparation involves having the patient drink a contrast agent, commonly known as x-ray dye, over a span of 2 hours, to allow for the contrast to uniformly coat the lining of the gastrointestinal tract (Prakashini et al. 2013). Completing the AXR before this oral preparation allows for some visualization of the patient's abdomen while the patient prepares for the ACT. There is minimally conclusive data provided from an AXR when the patient has abdominal pain or constipation, as evidenced

Table 1 Abdominal Imaging Data Collection for Nova Scotia Health Authority Hospitals.

\begin{tabular}{lcccc}
\hline Hospital & AXR & ACT & AXR and ACT & $\begin{array}{c}\text { Total abdominal } \\
\text { imaging examinations }\end{array}$ \\
\hline $\begin{array}{l}\text { Dartmouth General } \\
\text { Hospital }\end{array}$ & 86 & 144 & 30 & 230 \\
$\begin{array}{l}\text { Cobequid Community } \\
\text { Health Centre }\end{array}$ & 98 & 115 & 37 & 213 \\
Halifax Infirmary & 95 & 217 & 54 & 312 \\
\hline
\end{tabular}


by some of the reporting radiologist's. The reporting radiologist notes in the AXR report that the emergency room physician should refer to the ACT report, as it provides a better representation of the patient's condition. It seems that an AXR is a diagnostic exam that the emergency room physician has to obtain before they can order the ACT. As the ACT is ultimately performed, it can be investigated if it is necessary to request the AXR based on the diagnostic ability of both tests. This information can help provide the patient with a more accurate test and a more accurate diagnosis and treatment can be provided.

\section{DISCUSSION}

\section{Comparing Diagnostic Sensitivity of Abdominal Imaging Modalities}

The main goal of completing abdominal imaging exams is to provide significant information that allows for the emergency room physician or healthcare professional to make an informed diagnostic decision for the patient (Martin et al., 2015). Having an accurate imaging examination allows improved diagnostic accuracy of the patient's condition, promotes surgical planning and approach, speeds up the discharge or admission decision process, reduces hospital stays, and diminishes mortality and morbidity (Martin et al. 2015). A retrospective review by Kellow et al. (2008) characterized the utility of abdominal radiography for non-trauma emergency patients. Adults who underwent abdominal radiography over a 6 month period after presenting to the emergency department were included in this study. The specific institution used for this study permits the abdominal radiograph to be interpreted by the ordering physician, whose management decisions are made before a formal radiologic interpretation is provided by the radiologist (Kellow et al., 2008). Medical records were then reviewed to determine if the patient did or did not receive follow-up imaging, with the followup imaging consisting of ACT, abdominal ultrasound or an upper gastric study. A sub-analysis was performed to determine whether AXR was more sensitive for certain abnormalities (Kellow et al., 2008). Abdominal radiography was performed in 955 patients during the study period, with $50 \%$ of these patients undergoing further abdominal imaging; of those, CT was performed for $64 \%$ of patients. 
Of the $42 \%$ of patients who had normal AXR results, $72 \%$ were found to have abnormal findings on follow-up imaging (Kellow et al. 2008). Seventy-eight percent of patients with nonspecific results on the AXR received follow-up imaging (Kellow et al. 2008). In this study, follow-up imaging was performed more frequently for patients presenting with abdominal pain (55\%) and obstruction (49\%) (Kellow et al. 2008). Due to the large percentage of additional imaging after the normal AXR results, Kellow et al. (2008) concludes that emergency physicians are not sufficiently reassured by the lack of abnormal radiographic findings, as additional imaging is then revealing an abnormality not shown on AXR. AXR can be determined to have low diagnostic sensitivity, as AXR results that are nonspecific are then demonstrating abnormal results on other imaging (Kellow et al. 2008). AXR does not help the emergency room physician determine the cause of the patient's symptoms. It is recommended for patients who present to the emergency department with abdominal symptoms to skip the AXR and have more definitive imaging exams performed (Kellow et al. 2008). It has been concluded that AXR does not aid in the diagnosis of the patient's condition. Determining the most appropriate method of ACT imaging is also critical in correctly diagnosing patients.

\section{Standard-Dose CT vs. Low-Dose CT}

Haller, Karlsson \& Nyman (2010) evaluated whether a CT without the use of $\mathrm{x}$-ray dye provides more diagnostic information than AXR in patients with acute non-traumatic abdominal pain and if the use of $\mathrm{CT}$ can reduce the total number of additional radiographs the patient receives. A total of 222 patients received an AXR, standard-dose CT (SDCT) or low-dose CT (LDCT) from three time periods and were retrospectively reviewed (Haller, Karlsson \& Nyman 2010). For each of these patients, the indication for the exam was acute abdominal pain of unknown cause. The diagnosis in the AXR and ACT reports were compared with the diagnosis confirmed through operative or endoscopy methods. This comparison can help inform healthcare providers whether the imaging modality successfully diagnosed the associated etiology. From these comparisons, the percentage of ACT with correct diagnosis was significantly higher than the AXR. The SDCT and LDCT group were found to give a $50 \%$ correct diagnosis, compared to a $20 \%$ correct diagnosis provided with an AXR (Haller, Karlsson \& Nyman 2010). Surprisingly, 
LDCT was found to provide better diagnostic results than SDCT. Haller, Karlsson \& Nyman (2010) discuss that this is most likely due to radiologists increased experience in interpreting unenhanced ACT during the 2 years until LDCT was started.

\section{Low-Dose CT vs. AXR}

Nguyen et al. (2011) compared the use of LDCT and AXR in the primary investigation of acute abdominal pain and their diagnostic yield. Included in this study were patients with acute abdominal pain who would require an AXR through the emergency department and included patients during a 7 month period. These patients were randomized to either receive a LDCT or the standard AXR protocol. Of the 124 patients included in this trial, 60 received an AXR and 64 received an LDCT of the abdomen (Nguyen et al. 2011). A diagnosis was made in $12(21.8 \%)$ of patients who has an AXR, with the diagnosis's being bowel obstruction, fecal loading, and a pneumoperitoneum (Nguyen et al. 2011). After having an abdominal LDCT, 34 (64.2\%) patients received a diagnosis, with bowel obstruction, diverticulitis and acute pancreatitis being causes of the patients' symptoms (Nguyen et al. 2011). Patients were more likely to require further imaging investigations when AXR was performed $(50.9 \%$ of patients), whereas only 14 additional tests $(26.4 \%)$ were obtained when using the LDCT. These results indicate that LDCT provides sufficient diagnostic quality, producing a superior yield compared to AXR, with only a slight increase in radiation dose (Nguyen et al. 2011). This also concludes that the use of LDCT significantly reduces the number of subsequent imaging investigations during a patient's admission (Nguyen et al. 2011). An efficient and accurate diagnosis through one diagnostic imaging exam reduces the use of other diagnostic tests, allowing for radiation dose to be reduced.

\section{Effective Radiation Dose}

Many physicians rely on AXR as a first diagnostic imaging modality as it is widely available and has a lower radiation exposure than ACT (Gans, Stoker, \& Boermeester 2012). As ionizing radiation is used in both AXR and ACT imaging, radiation dose must be considered when determining the appropriate exam as it can have harmful effects to the body if. According to Health Canada and Safety Code 35 (2008), the yearly radiation dose an individual should receive is an effective dose of $1 \mathrm{mSv}$. Effective dose is the 
measure of the total risk due to an exposure to ionizing radiation (Canadian Nuclear Safety Commission 2019). AXR typically results in an effective dose of $0.7 \mathrm{mSv}$ of radiation, whereas a standard-dose ACT produces roughly $10 \mathrm{mSv}$ of radiation (Gans, Stoker, \& Boermeester 2012). Although the ACT exceeds the dose limit, $10 \mathrm{mSv}$ is considered a reasonable radiation dose when used in moderation with appropriate dose reduction methods, as someone must receive close to $1,000 \mathrm{mSv}$ to have radiation sickness symptoms (Canadian Nuclear Safety Commission 2019). As evidenced by data collection, patients are receiving two diagnostic imaging exams when presenting to the emergency department for abdominal symptoms in these Nova Scotia facilities, which leads to a higher total radiation dose. As the ACT is ultimately performed in these cases due to its high diagnostic ability, it is worthwhile noting that that by eliminating a test, a reduction in the patient's overall radiation dose can be made.

\section{Methods for Reducing Radiation Dose in Abdominal Imaging}

Strategies can be used to reduce the dose while maintaining the diagnostic image quality that it provides. Haller, Karlsson \& Nyman (2010) suggests reducing the x-ray current and time product from 120-260 mAs to 30-76 mAs to generate a LDCT. Fewer x-rays will be emitted over a period of time, resulting in a reduction of radiation that the patient receives. Previous discussion states that LDCT is diagnostically sufficient, even with the reduction of radiation. The effective dose of a LDCT of the abdomen is approximately $4.2 \mathrm{mSv}$, which is still higher than an AXR, but is approximately a $58 \%$ reduction of dose in comparison to using a SDCT (Haller, Karlsson \& Nyman 2010). Alshamari et al., (2016) declared even lower dose calculations for AXR and LDCT of the abdomen. During their study, the AXR resulted in an effective dose of $1.0 \mathrm{mSv}$, while the low-dose ACT provided an effective dose of $1.2 \mathrm{mSv}$ (Alshamari et al., 2016). This lower dose level is sufficient for CT to detect fluidfilled dilated bowel and free intra-abdominal gas (Alshamari et al. 2016), deeming it diagnostically capable at a lower radiation dose to the patient. This demonstrates how creating a low-dose protocol on hospital CT scanners to be used for these specific emergency room cases is optimal as the ACT provides more information than the AXR and is performed at a low enough dose that it does not significantly impact the patient. 


\section{Adaptive Statistical Iterative Reconstruction vs. Filtered Back Projection}

ASIR is a technique that can be used to decrease radiation dose. A reduction in image quality called noise can be evident when a reduction of radiation is used, but ASIR can help to reduce image noise by using a statistical reconstruction algorithm (Sagara et al. 2010). Sagara et al. (2010) completed a study to compare image quality and dose for patients who either received a LDCT reconstructed with ASIR or a SDCT reconstructed with filtered back projection (FBP). The study group for this article comprised 169 LDCT scans using ASIR, with 53 of these 169 patients previously having a SDCT that used FBP. The volume CT dose index $\left(\mathrm{CTDI}_{\mathrm{vol}}\right)$, a standardized measurement of radiation for a $\mathrm{CT}$ scanner, was obtained for LDCT with ASIR and SDCT with FBP by using PACS. The LDCT with ASIR averaged $17 \mathrm{mGy} \mathrm{CTDI}_{\text {vol }}$ compared to $25 \mathrm{mGy}$ CTDIvol for the SDCT with FBP, providing a 33\% dose reduction by using LDCT with ASIR (Sagara et al. 2010). In regards to effective dose, $13 \mathrm{mSv}$ is provided for LDCT compared to $18 \mathrm{mSv}$ for SDCT (Sagara et al. 2010). The LDCT has a significantly less amount of image noise as compared to the SDCT (see Figs 3 and 4), demonstrating that the LDCT with ASIR does not reduce diagnostic acceptability and maintains the diagnostic quality at a lower dose (Sagara et al., 2010).

\section{CONCLUSION}

Abdominal imaging is frequently requested by emergency departments to determine the cause of a patient's abdominal pain. In three multiple Nova Scotia hospitals, it is common for both an AXR and ACT to be ordered for these patients. Research shows that an ACT demonstrates higher diagnostic quality than an AXR and is ultimately ordered. Imaging techniques, such as LDCT and ASIR, can reduce the radiation dose of an ACT while maintaining diagnostic quality. The results of this research demonstrate the need for increased education on proper exam ordering practice for AXR versus ACT. Emergency departments should be encouraged to develop appropriate imaging protocols when patients present with abdominal pain. This will impact the ordering practice and reduce radiation dose by avoiding additional imaging. More research into this topic will help determine the cost differences between an AXR 


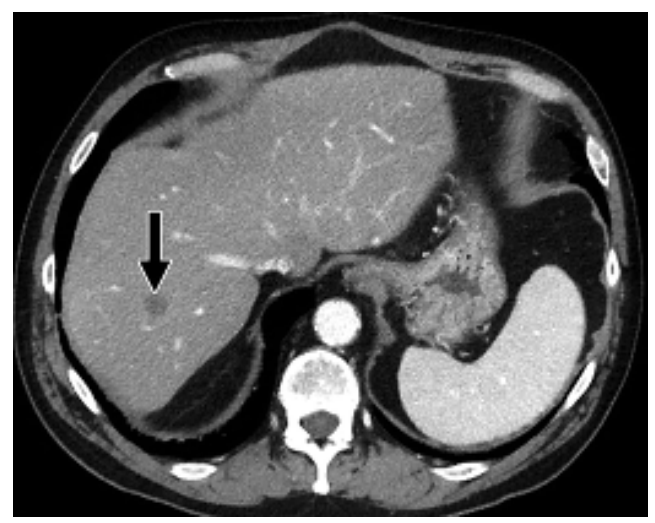

Fig 3 LDCT with ASIR (Sagara et al. 2010).

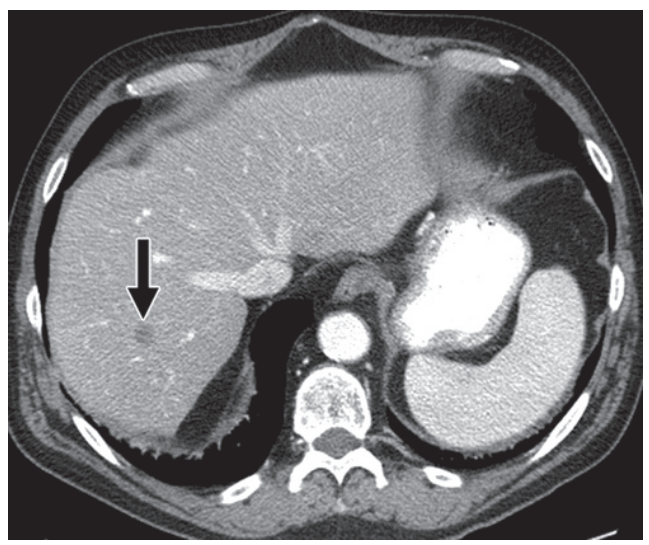

Fig 4 SDCT with FBP (Sagara et al. 2010).

and ACT and the impact with this protocol change could have on the workload of the emergency and diagnostic imaging departments.

Acknowledgements I acknowledge that this writing is wholly my own. I would like to thank my instructor, Rachel MacLeod, for assisting in editing this paper, Stephanie Schofield for the inspiration to research this topic and Dr. A. Costa for guidance and mentorship discussion. 


\section{REFERENCES}

Alshamari, M., Norrman, E., Geijer, M., Jansson, K. \& Geijer, H. (2016). Diagnostic accuracy of low-dose CT compared with abdominal radiography in non-traumatic acute abdominal pain: prospective study and systemic review. European Radiology 26(6): 1766-1774. doi:10.1007/ s00330-015-3984-9.

Bertin, C., Ponthus, S., Vivekanantham, H., Poletti, P-A., Kherad, O. \& Rutschmann, O. (2019). Overuse of plain abdominal radiography in emergency departments: a restrospective cohort study. BMC Health Services Research 19: 36. doi.org/10.1186/s12913-019-3870-2.

Canadian Associations of Radiologists. (2012). Diagnostic Imaging Referral Guidelines. Retrieved from: car.ca/patient-care/referral-guidelines/

Canadian Nuclear Safety Commission. (2019). Radiation doses. Retrieved from the Government of Canada website: nuclearsafety.gc.ca/eng/resources/radiation/introduction-to-radiation/ radiation-doses.cfm.

Cartwright, S. \& Knudson, M. (2015). Diagnostic Imaging of Acute Abdominal Pain in Adults. American Family Physician 91(7): 452-459. Retrieved from: www.aafp.org/afp/2015/0401/p452.html.

Gans, S., Stoker, J. \& Boermeester, M. (2012). Plain abdominal radiography in acute abdominal pain; past, present and future. International Journal of General Medicine 12(5): 525-533. doi:10.2147/IJGM.S17410.

Haller, O., Karlsson, L. \& Nyman, R. (2010). Can low-dose abdominal CT replace abdominal plain film in evaluation of acute abdominal pain? Upsala Journal of Medical Sciences 115: 113-120. doi:10.3109/03009730903294871.

Health Canada. (2008). Safety Code 35: Procedures for the Installation, Use and Control of X-ray Equipment in Large Medical Radiological Facilities. Retrieved from the Government of Canada website:

www.canada.ca/en/health-canada/services/environmental-workplacehealth/reports-publications/radiation/safety-code-35-safety-proceduresinstallation-use-control-equipment-large-medical-radiological-facilitiessafety-code.html.

Kellow, Z., MacInnes, M., Kurzencwyg, D., Rawal, S., Jaffer, R., Kovacina, B. \& Stein, L. (2008). The Role of Abdominal Radiography in the Evaluation of the Nontrauma Emergency Patient. Radiology 248(3): 887-893. doi:10.1148/radiol.2483071772.

Martin, J., de Gracia, M., Torres, C., Martinez, D. \& Herranz, P. (2015). Routine abdominal x-rays in the emergency department: A thing of the past? Radiologia 57(5): 380-390. doi:doi.org/10.1016/j.rxeng.2015.08.001.

Nguyen, L., Wong, D., Fatovich, D., Yeung, J., Persaud, J., Wood, C., de Vos, D. \& Mendelson, R. (2011). Low-dose computed tomography versus plain abdominal radiography in the investigation of an acute abdomen. ANZ Journal of Surgery 82(1-2). doi:doi.org/10.1111/j.1445-2197. 2010.05632.x. 
Nova Scotia Health Authority. (2019). CT Protocols and Policies.

Picture Archive Computer System (PACS). (2019). Retrieval date: October 20 ${ }^{\text {th }}, 2019$.

Prakashini, K., Kakkar, C., Sambhaji, C., Shetty, C.M. \& Rao, V.R. (2013). Quantitative and qualitative bowel analysis using mannitol, water and iodine-based endoluminal contrast agent on 64-row detector CT. The Indian Journal of Radiology \& Imaging 23(4): 373-378. doi:10.4103/0971-3026.125594.

Sagara, Y., Hara, A., Pavlicek, W., Silva, A., Paden, R. \& Wu, Q. (2010). Abdominal CT: Comparison of Low-Dose CT with Adaptive Statistical Iterative Reconstruction and Routine-Dose CT with Filtered Back Projection in 53 Patients. Medical Physics and Informatics 195. doi:10.2214/ AJR.09.2989. 
